\title{
Schultheoretische Anfragen zum pädagogisch-normativen Heterogenitätsdiskurs
}

\section{Beate Wischer und Matthias Trautmann}

Der Beitrag zielt auf eine kritische Auseinandersetzung mit pädagogisch-normativen Reflexionen im aktuellen Heterogenitätsdiskurs. Rekonstruiert wird zunächst der hier zugrunde liegende Reflexionstyp, um davon ausgehend drei ausgewählte Probleme zu diskutieren: (1) eine Unschärfe und Überkomplexität des Heterogenitätsbegriffs, der aus einem Denken vom einzelnen Kind resultiert, (2) eine Ausblendung der Struktur- und Funktionslogiken institutionalisierten Lernens und (3) professionstheoretische Verkürzungen durch normativ-pädagogische Lehrerleitbilder.

\section{Problemaufwurf}

«In den öffentlichen Debatten» - so stellte unlängst Bernd Zymek (2011, S. 83) fest - «haben wir Pädagogen es erreicht, dass das Thema ,Homogenität und Heterogenität im Schulsystem' als ein pädagogisches und psychologisches behandelt» werde: «Wir haben es geschafft, unsere Sicht der Dinge durchzusetzen (also alles «vom Kinde aus», als Problem der Unterrichtsorganisation und der Methodik zu thematisieren) und diese Sicht des Themas als die einzige legitime im öffentlichen Diskurs durchzusetzen». Zymek bezeichnet diese Dominanz einer pädagogischen Perspektive zwar als einen «professionellen Erfolg». In seiner Analyse überwiegen jedoch die kritischen Untertöne: Das Problemfeld rund um Heterogenität bzw. Homogenität im Schulsystem ist - so zeigen seine Analysen zur Historie und zu aktuellen Transformationsprozessen im Bildungssystem - erheblich komplexer und widersprüchlicher als dies die in der aktuellen Diskussion doch oft anzutreffenden (pädagogischen) Problembeschreibungen und Reformideen abzubilden vermögen.

Freilich lässt sich die kritische Diagnose von Zymek keinesfalls auf die Gesamtheit der Auseinandersetzungen zur Heterogenitätsthematik übertragen. Man hat es mit überaus komplexen Problemstellungen zu tun, an deren Bearbeitung - und das oft schon seit Jahrzehnten - diverse schulpädagogische Teildisziplinen (z.B. Allgemeine Didaktik, empirische Bildungsforschung, 
Schul- und Professionstheorie), aber auch ausserdisziplinäre Forschungsfelder (z.B. psychologische Lehr-Lern-Forschung, soziologische Ungleichheitstheorie) direkt und indirekt beteiligt sind. Entsprechend gross ist zudem die Bandbreite der Publikationen in Bezug auf deren Anlage und Anspruch - das Spektrum reicht von ambitionierten theoretischen und empirischen Analysen über Erfahrungsberichte und programmatische Beiträge bis hin zu einer auf die Praxis orientierten (Ratgeber-)Literatur (vgl. zusf. Wischer, 2009). Und zu berücksichtigen sind selbstverständlich auch länderspezifische Problemlagen und Diskurse.

Allerdings ist auch nicht von der Hand zu weisen, dass man es bei dem Thema aktuell mit einem Reformthema ersten Ranges zu tun hat. Der bisherige Umgang mit Heterogenität (nicht allein im deutschen) Schulsystem wird in der Regel nicht nur als problematisch markiert, sondern man trifft auch auf umfassende Empfehlungen, Programme und Konzeptideen, die eine Verbesserung, wenn nicht gar eine Beseitigung der Problemlagen versprechen (z.B. Bräu \& Schwerdt, 2005; Buholzer \& Kummer-Weiss, 2010). Betrachtet man diese gleichsam auf Reformen zielenden Beiträge genauer, dann zeichnet sich ausserdem ab, dass zumindest hier - wie von Zymek kritisiert - eine von pädagogischen Prämissen geleitete Problembeschreibung und -behandlung durchaus recht weit verbreitet ist: Viele programmatische Beiträge zum aktuellen Heterogenitätsdiskurs - so lautet deshalb unsere Ausgangsthese - reflektieren die Thematik zuvorderst aus einer pädagogisch-normativen Perspektive, von einer ,Pädagogik vom Kinde aus', mit dem Ergebnis einer verkürzten Problemsicht auf das Bedingungsgefüge organisierter Bildungs- und Erziehungsprozesse.

Zur Entfaltung dieser These rekonstruieren wir zunächst den pädagogisch-normativen Reflexionstyp in seinen zentralen Merkmalen (Abschnitt 2), um davon ausgehend drei ausgewählte Problembereiche aus einer schultheoretischen Perspektive in den Blick zu nehmen: die Überkomplexität und Unschärfen des Heterogenitätsbegriffs (3), die Ausblendung institutioneller Rahmenbedingungen schulischen Lernens (4) und professionstheoretische Verkürzungen, die mit einer Fokussierung auf berufsethische Dimensionen einhergehen (5). Wir enden mit der Frage, was man gewinnt (und was man verliert), wenn man sich auch unter Reformgesichtspunkten auf eine stärker analytisch-distanzierte bzw. konkret: auf eine schul- und organisationstheoretische Perspektive einlässt.

\section{Pädagogisch-normative Reflexionen im aktuellen Heterogenitätsdiskurs}

Der Ausgangspunkt unserer Überlegungen wird durch zwei Probleme markiert, die keineswegs neu sind: Zuerst einmal wird über die Frage nach dem Umgang der Schule mit der Unterschiedlichkeit ihrer Adressaten selbst eine Thematik angesprochen, die pädagogische Reflexionen seit jeher sehr intensiv beschäftigt hat (vgl. schon Trapp, 1780). Denn es geht im Kern um konstitutive Probleme 
der modernen Schule bzw. um Herausforderungen, die mit institutionalisierten und organisierten Bildungsprozessen unweigerlich verknüpft sind: Findet Lernen nicht mit Einzelnen (wie im Hauslehrermodell früherer Zeiten), sondern in grösseren Gruppen statt, dann stellt sich - so kann man dies etwas salopp konkretisieren - automatisch die Frage ein, wie ein solcher Gruppenunterricht realisiert werden kann, ohne dabei die Bedürfnisse der Einzelnen zu vernachlässigen. Das zweite Problem steckt in den disziplinären Zugangsweisen zu solchen (und anderen) Fragen: Erziehungswissenschaftliche resp. pädagogische Reflexionen - so verkürzt zusammengefasst (ausf. Tenorth, 1987) - zielen in der Regel nicht nur auf eine distanzierte Analyse der pädagogischen Praxis, mithin nur auf Erkenntnisgewinn. Es gibt zumindest in Deutschland auch einen überaus traditionsreichen Anspruch, gleichsam als «Anwalt für das Kind» für dessen Eigenrecht einzutreten und davon ausgehend normative Vorgaben wie auch Konzepte für eine an den Bedürfnissen der lernenden Subjekte ausgerichteten Schule zu entwickeln (z.B. Montessori, 1916/1976; Bohnsack, 2008). Verbunden ist dies mit einem spezifischen Modus der Reflexion, den man in Anlehnung an Luhmann und Schorr (1988) auch als Reformreflexion bezeichnen könnte und der sich unmittelbar bezogen auf die Heterogenitätsthematik - vereinfacht über folgende Figur charakterisieren lässt:

- Etablierung einer Norm/eines Massstabs: Unter der zentralen Prämisse, dass Kinder und Jugendliche als einzigartige Subjekte und ganze Persönlichkeiten wahr- und anzunehmen sind, wird Heterogenität nicht nur als der Normalfall einer jeden Lerngruppe betrachtet, sondern als ein grundsätzlich positiver Wert aufgestellt (z.B. Buholzer \& Kummer-Wyss, 2010, S. 10). Unterschiede zwischen den Mitgliedern einer Lerngruppe (als Resultat der vielen einzigartigen Persönlichkeiten) dürfen weder als Problem bewertet noch ignoriert, eingeschränkt oder abgebaut werden (dies entspräche einer Missachtung und Normierung), sondern sie seien als Chance und Bereicherung zu begreifen und produktiv zu nutzen: Jedes Kind habe ein Recht auf optimale individuelle Förderung und Unterstützung.

- Schul-und Unterrichtskritik: Als Kontrastfolie wird das Bild einer Schulpraxis gezeichnet, die Heterogenität auf vielfältige Weise ignoriere und reduziere, und individuelle Schülerbedürfnisse grundlegend missachte. In der Kritik stehen normierte Lehr-Lern-Prozesse (durch gleichförmigen ,Frontalunterricht' und einheitliche Lehrplanvorgaben), die Klassifikation von Schülerinnen und Schülern bzw. deren kategoriale (und nicht partikularistische) Behandlung ebenso wie sämtliche Formen der Homogenisierung (von Lerngruppen, Schülerschaften), die auf eine Reduzierung von Vielfalt zielen und mit Ausschluss, mit «immer neuen Schritten der Selektion verbunden» seien (Tillmann, 2008, S. 63). 
- Programmatik: Von dieser Differenz ausgehend werden zahlreiche Forderungen abgeleitet, um die Lücke zwischen dem Ideal und der Realität zu schliessen. Gefordert werden Veränderungen der Organisation bzw. des Systems wie Abschaffung des gegliederten Schulsystems und ein Verzicht auf externe Leistungsdifferenzierung und Selektion (z.B. Ratzki, 2005). Es gibt aber vor allem auch vielfältige, auf die Interaktionsebene gerichtete Reformideen für eine heterogenitätssensible Lern- und Förderkultur und für einen wertschätzenden Umgang mit Vielfalt (z.B. Bräu \& Schwerdt, 2005; Grossrieder, 2010): Nicht die Lernenden - so die programmatische Stossrichtung - müssten sich der Schule, sondern die Schule bzw. die in ihr tätigen Erwachsenen müssten sich dem einzelnen Kind anpassen, müssten dessen je individuellen Bedürfnissen gerecht werden (Schäfers, 2009, S. 42).

Nun soll weder in Zweifel gezogen werden, dass bisherige Strategien im Umgang mit Schülerheterogenität oft problematisch und verbesserungsbedürftig sind; noch wird in Abrede gestellt, dass mit pädagogisch-normativen Prämissen durchaus notwendige Orientierungsmarken für pädagogisches Handeln und für Schulgestaltung gesetzt werden. Probleme sehen wir jedoch in Bezug auf diesen spezifischen Modus der pädagogischen Argumentation und Reflexion, der - wie wir über drei kritische Anfragen zeigen wollen - aus einem schul- und organisationstheoretischen Blickwinkel mit erheblichen Verkürzungen verbunden ist. Gestaltungsspielräume für Reformen - so die dahinter stehende Überlegung lassen sich nur dann erfolgversprechend ausloten, wenn man das komplizierte und komplexe Bedingungsgefüge institutionalisierter Lernprozesse in all seinen Facetten und Widrigkeiten einbezieht.

\section{Die Unschärfen des Heterogenitätsbegriffs - erste schultheoretische Anfrage}

Heterogenität ist zwar ein Schlüsselbegriff, unter dem derzeit vielfältige schulpädagogische Analysen und Reformideen firmieren; damit verbunden sind aber - und dies ist zunächst ein grundsätzliches Problem - bislang weder ein einheitliches theoretisches Konzept noch konsistente Programmideen. Es gibt vielmehr ein Nebeneinander von differenten Zugangsweisen mit unterschiedlichem Fokus (Budde, 2012; Bohl, Patzel \& Richey, 2012) - und je spezifischen Chancen und Schwierigkeiten. So problematisieren sozialwissenschaftliche Diskurse die Konstruktionsprinzipien von Differenzlinien (wie Geschlecht, Nationalität oder Behinderung) und damit verbundene Ungleichheiten: Unterschiede gelten als historisch und gesellschaftlich bedingte Konstruktionen, in die Vorstellungen von Normalität und Abweichung, Dominanz, Hierarchie und Unterdrückung eingebaut sind, wobei die Schule hier als eine Institution in den Blick gerät, die an der Herstellung gesellschaftlicher Ungleichheiten beteiligt ist. Differenzen und deren Ungleichheitsrelationen müssen demnach zwar wahrgenommen werden, gewarnt wird aber vor der Gefahr einer Dramatisierung und Essentialisierung 
(im Überblick Sauter \& Schröder, 2007). Einen gänzlich anderen Zugang bietet die psychologische Lehr-Lern-Forschung, die Lernermerkmale (Vorwissen, kognitive Grundfähigkeiten, aber auch motivationale und affektive Dimensionen) als Bedingungsfaktoren schulischer Leistungen modelliert (Helmke \& Weinert, 1997). Unter schultheoretischen Gesichtspunkten wird so einmal ein deutlich engeres Mandat der Schule (effektive Förderung fachlicher Lernleistungen) und vorrangig nur eine Berücksichtigung von lern- und leistungsbezogenen Lernermerkmalen (und kaum von sozialstrukturellen Kategorien) fokussiert. Zum anderen fehlt weitgehend eine Problematisierung bezüglich einer Essentialisierung oder Ontologisierung von Lernermerkmalen, obwohl z.B. das (umstrittene) Intelligenzkriterium zu einer solchen Reflexion geradezu einlädt.

In den pädagogisch-normativen Reflexionen, die uns hier beschäftigen, findet man nun zwar durchaus einen Rückgriff auf diese beiden recht unterschiedlichen Zugangsweisen zum Heterogenitätsproblem (Kampshoff, 2009, S. 37): Es werden individuelle, leistungsbezogene Lernervoraussetzungen und soziale Gruppenzugehörigkeiten genannt, dies allerdings ohne eine systematische Auseinandersetzung mit den damit jeweils verbundenen theoretischen Prämissen. Man trifft stattdessen - zugespitzt formuliert - auf lange und beliebig erweiterbare Auflistungen von Merkmalen, in denen sich die Mitglieder einer Lerngruppe unterscheiden können (z.B. Altrichter \& Hauser, 2007; Höhmann, 2009), und die ungewichtet als bedeutsam eingeschätzt werden: Konstitutive Basis zur Bestimmung von Heterogenität sind die einzigartigen Persönlichkeiten (als ganze Personen) mit der Folge einer begrüssenswerten (und zu bewahrenden) Vielfalt in theoretisch infiniten Dimensionen.

Wie wir nun über einige kritische Anfragen andeuten wollen (Trautmann \& Wischer, 2011, Kap. 2; Emmerich \& Hormel, 2013, Kap. II. 4), fungiert der Heterogenitätsbegriff in einer solchen Verwendung doch eher als ein Slogan, der sich zwar als Reformformel als anschlussfähig erweist, die dahinter stehenden Probleme und Herausforderungen aber eher verdeckt als aufklärt:

- Über die Referenz auf die Einzigartigkeit der Subjekte wird Heterogenität zu einer überkomplexen und gleichzeitig auch inhaltsleeren Grösse: Welche Unterschiede gemeint sind, bleibt letztlich offen bzw. kann um beliebig viele Dimensionen erweitert werden. Verkannt (oder unterschätzt) werden damit nicht nur kapazitäre Begrenzungen - in der Wahrnehmungsfähigkeit der Akteure, aber auch in den Verarbeitungsmöglichkeiten der Schule als Organisation (s.u.). Es fehlt auch an einer analytischen Tiefenschärfe. Dies beginnt mit einer unzureichenden Klärung der epistemologischen Prämissen: Werden Unterschiede bzw. Merkmale als ontologische, sozialstrukturelle oder als beobachterabhängige Grössen gefasst? Dazu gehört aber auch eine aus schultheoretischem Blickwinkel diffuse Kontextualisierung und Zielreflexion: Die Vielfalt an Dimensionen und deren explizite Nichtgewichtung verweisen zwar auf ein über fachliches Lernen deutlich hinausgehendes Mandat der 
Schule. Unklar bleibt aber z.B. das Verhältnis differenter Funktionen und Aufgaben der Schule und deren Implikationen für die Wahrnehmung und Berücksichtigung einzelner Heterogenitätsdimensionen: Unter welchen Bedingungen sind welche Kriterien bedeutsam und mit welcher Zielstellung? Wozu dienen die umfänglich einbezogenen askriptiven Merkmale? Gibt es auch legitime Homogenitätserfordernisse?

- Zwar basieren pädagogische Konzeptionalisierungen auf einem weiten Mandat der Schule, dies schliesst jedoch nicht - wie in den sozialwissenschaftlichen Differenzdiskursen - eine systematische Reflexion gesellschaftlicher und institutioneller Ungleichheitsformen mit ein. Der pädagogische Blick auf das Individuum (dem es traditionell an Gesellschaftstheorie fehlt) wie auch die enge Bindung an positive Wertungsoptionen (das charakteristische ,Feiern' von Vielfalt) führen vielmehr dazu, die faktischen Ungleichheiten von Personen, Kriterien oder Positionen auszublenden. Dies wirft kritische Rückfragen dann auch für die konkrete Förderung auf: Wenn Unterschiede im individuellen Entwicklungsstand oder den Kompetenzen (z.B. Lesekompetenz) im Extremfall nur als Zeichen von Vielfalt, nicht aber auch als Ausdruck von noch nicht realisierten Entwicklungspotenzialen (also als Defizite) bewertet werden dürfen, dann fehlt es im Prinzip an Maßstäben, um überhaupt fördern und problematische Unterschiede dann auch beseitigen zu können.

- Wie Emmerich \& Hormel (2013, Kap. II. 4) überzeugend herausarbeiten, läuft der pädagogisch-normativ konturierte Heterogenitätsbegriff nicht zuletzt wegen seiner theoretischen Unschärfen auf eine Ontologisierung und Essentialisierung von Heterogenitätsdimensionen einerseits und auf eine erhöhte Bedeutung askriptiver Merkmale andererseits hinaus. Indem Individualität als im Kind angelegt und in das Klassenzimmer mitgebracht vorgestellt wird, gerät einmal die Hervorbringung von Unterschiedlichkeit durch die Institution Schule selbst ausser Blick (vgl. auch Budde, 2012). Zugleich werden über Forderungen nach einer umfassenden Behandlung der Lernenden als ganze Personen - etwa bei der Diagnostik - zugeschriebene, nicht durch eigene Leistung veränderbare Merkmale wie Geschlecht, soziale oder ethnische Herkunft ganz explizit in das Bewusstsein der Akteure gerückt - mit der möglichen Folge, dass darüber entgegen der pädagogischen Absichten problematische Klassifizierungen und Stereotypisierungen gerade erst befördert werden könnten.

\section{Die Ausblendung institutioneller Bedingungen - zweite schultheoretische Anfrage}

Die bislang verhandelten Probleme lassen sich schärfen, wenn man den Kontext einbezieht, in dem das schulische Lernen stattfindet. Im Prinzip liegt es auf der Hand und hatten wir schon angedeutet: Aus einer pädagogisch-normativen 
Perspektive, d.h. vom einzelnen Subjekt ausgehend gedacht, muss die Organisation von Massenlernprozessen in institutionalisierten und formalisierten Systemzusammenhängen (dem Schul- bzw. Bildungssystem) eine vehemente Schulkritik geradezu herausfordern. Anders als im Hauslehrermodell oder bei informellen Lernanlässen ist hier eine Missachtung individueller Lernerbedürfnisse strukturell eingelassen und werden Einflussgrössen virulent, die von pädagogischen Ideen und Motiven abstrahieren bzw. diesen sogar entgegen stehen. Man kann auch zugespitzt argumentieren: Die Frage, wie Schule mit der Unterschiedlichkeit ihrer Adressaten umgehen kann und soll, markiert nicht nur ein konstitutives Problem der modernen Schule, sondern sie musste auch zu einem zentralen Bezugsproblem pädagogisch-normativer Reflexionen werden (im Folgenden ausf. auch Wischer, 2013).

Die Referenz für diese Zuspitzung liegt in den Merkmalen und Ambivalenzen der Institutionalisierung (Leschinsky \& Cortina, 2005), die die Pädagogik in Deutschland von Beginn an herausgefordert und belastet haben (z.B. Terhart, 1986): Nur durch Institutionalisierung - das ist die eine Seite des Problems kann ein flächendeckendes Bildungsangebot überhaupt erst sicher und auf Dauer gestellt werden. Damit verknüpft sind allerdings organisatorische Prämissen, die mit pädagogischen Prämissen in Konflikt geraten (können). Das gilt einmal für organisationsspezifische Modalitäten bezüglich des Umgangs mit Heterogenität: Es sind grosse Schülerströme zu kanalisieren, Laufbahnen zu strukturieren und Übergänge wie auch Gruppenzugehörigkeiten (Mitgliedschaftsbedingungen) verbindlich zu regeln, was Vereinheitlichungsstrategien voraussetzt und eine kategoriale (und nicht partikularistische) Schülerbehandlung nahe legt (Leschinsky \& Cortina, 2005). Organisationen - so lässt sich das Problem in organisationssoziologischer Perspektive zuspitzen - zeichnen sich dadurch aus, dass sie vom Einzelfall und von den konkreten Subjekten abstrahieren und stattdessen eine «Vielzahl von individuellen Bedürfnissen, Wünschen und Problemlagen bündeln und typisieren und dann nach demselben Schema abarbeiten» (Preisendörfer, 2008, S. 161). Weitere Probleme für eine allein an pädagogischen Ideen orientierte Schulgestaltung entstehen durch die «Grammatik der Institutionalisierung» (Leschinsky \& Cortina, 2005, S. 28). Dazu gehört etwa die historisch gewachsene Einbindung des Bildungssystems in gesellschaftliche Funktionszusammenhänge (z.B. Klafki, 2002), in deren Folge es in der Schule nicht nur um optimale Förderung und individuelle Entwicklung geht. Es gibt auch Standards für Qualifizierungen und individuelle Lernerfolge/-misserfolge werden über Zensuren/Schulabschlüsse karrierewirksam markiert. Daneben scheinen Institutionalisierungsprozesse und organisationales Handeln aber auch eigene Logiken, Rationalitäten und Dynamiken zu entfalten, denen man weder durch moralische Appelle noch durch politische Eingriffe leichthin beikommen kann (ebd., auch Gomolla \& Radtke, 2003).

Es sind nicht zuletzt solche Imperative der Institutionalisierung, die eine pädagogische Kritik an der Schule seit jeher aktiviert (Diederich \& Tenorth, 
1997) und im Gegenzug eine zu den Systemelementen und Funktionsprinzipien der herkömmlichen Schule komplementäre Programmatik befördert haben (Luhmann \& Schorr, 1988). Das ist die andere Seite des Problems: In pädagogisch-normativen Reflexionen wird der Organisationsfaktor weniger als eine strukturell notwendige Grundbedingung schulischen Lernens gefasst, um darüber die Spielräume (und Grenzen!) für pädagogisches Handeln in Organisationen in den Blick zu bekommen. Vielmehr gibt es eine lange pädagogische Tradition von Abgrenzungen, Frontstellungen und Ausblendungen (Luhmann \& Schorr, 1988; Terhart, 1986), in die sich nach unserem Dafürhalten auch die pädagogisch-normativen Reflexionen im aktuellen Heterogenitätsdiskurs einordnen lassen.

Zentrale Struktur- und Funktionsprinzipien organisierter Bildung geraten vor allem über zwei Problembereiche in den Blick. Der erste Bereich betrifft die (organisatorisch notwendigen) Vereinheitlichungsstrategien und Normierungsprozesse, die mit dem Anspruch des Kindes auf individuelle Zuwendung und Förderung konfligieren. Die Kritik richtet sich hier auf normierte Schülerbehandlungen auf der Interaktionsebene (den gleichschrittigen ,Frontalunterricht') wie auch auf organisatorische Prinzipien der (kriteriengeleiteten) Lerngruppenkonstituierung und Schulformdifferenzierung. Während man im ersten Fall jedoch ein Mehr an (innerer) Differenzierung und Individualisierung proklamiert, laufen die Forderungen in Bezug auf die Organisationsebene im Kern auf einen Verzicht auf (äussere) Differenzierung hinaus (z.B. Ratzki, 2005; Tillmann, 2008). Als zweite Störgrösse fungieren Selektionsentscheidungen, die über die Beteiligung der Schule an gesellschaftlicher Allokation folgenreich sind, auf operativer Ebene aber auch zu permanenten «Besser-Schlechter-Klassifikationen» und im Ergebnis zu einer, ungleichwertigen Verschiedenheit' führen. Unterschiede - so dazu plakativ die pädagogischen Prämissen - sollen in der Schule nicht als besser oder schlechter bewertet, sondern anerkannt werden. Und es darf nur um Förderung gehen, nicht aber um Selektion!

Dass ein solcher Umgang mit organisatorischen Prämissen deutlich zu kurz greifen muss, ist in unseren Ausführungen als Argument im Prinzip bereits angelegt. Wir versuchen die hier auftauchenden Probleme über drei Beispiele zu plausibilisieren (Trautmann \& Wischer, 2011, Kap. 3; Wischer, 2009):

Ein grundsätzliches Problem sehen wir darin, dass die strukturellen und funktionellen Elemente inklusive deren komplexen Verflechtungen, auch zum Gesellschaftssystem, kaum systematisch in den Blick genommen werden. So stehen zwar einzelne Organisationselemente der Schule - wie die Homogenisierung von Lerngruppen - im Zentrum der Kritik. Diese werden aber weniger in ihren multiplen, auch ausserpädagogischen Funktionen reflektiert (für Lerngruppendifferenzierung vgl. schon Keim, 1977), sondern nahezu ausschliesslich in ihren Folgen für die Schülersubjekte problematisiert. Zudem fehlt es oft an einer Verknüpfung der unterschiedlichen Handlungsebenen des Bildungssystems, d.h. an einer mehrebenenanalytischen Betrachtung (Fend, 
2008). Handlungsempfehlungen richten sich überwiegend auf die Mikroebene - auf didaktisch-methodische Unterrichtsgestaltung und Diagnostik (z.B. Bräu $\&$ Schwerdt, 2005). Unhinterfragt bleiben jedoch die Vorgaben und Einflüsse der Meso- und Makroebene: Sind überhaupt die notwendigen Voraussetzungen vorhanden? Welche strukturellen Bedingungen rahmen die Unterrichtsprozesse und Interaktionsformen?

Die Verlagerung der Problemlösungen von der Organisations- auf die Interaktionsebene eröffnet gleichzeitig - so lässt sich mit Luhmann und Schorr (1988) argumentieren - Optionen, um unliebsamen Fragen und Problemen auszuweichen, die mit gesellschaftlich organisierter Erziehung (und so auch mit der Heterogenitätsthematik) unweigerlich verknüpft sind. Zwei weitere Anfragen sollen dies zumindest andeuten (vgl. ausf. Wischer, 2013; Emmerich \& Hormel, 2013):

- Eine Anfrage betrifft Ziele von Förder- bzw. auch Differenzierungsstrategien, die angesichts der multiplen Funktionen moderner Bildungssysteme nicht nur vielschichtig sind, sondern auch in zahlreiche Konflikte und Dilemmata führen: Geht es z.B. um optimale individuelle Leistungsentwicklung oder (auch) um den Ausgleich interindividueller Leistungsunterschiede? Bei ,äusserer' (organisatorischer) Differenzierung werden derartige Entscheidungsprobleme sehr konkret sichtbar, weil hier organisationale, mithin universell gültige Zugangs- und Exit-Regeln vorhanden sein müssen und weil Entscheidungen (etwa bezüglich der Zugehörigkeit zu einer Lerngruppe) in der Regel über einen längeren Zeitraum Gültigkeit haben. Formen von ,innerer' Differenzierung (wie sie der Reformdiskurs präferiert) sind demgegenüber zwar flexibler (Ziele oder Schülergruppierungen können etwa von Stunde zu Stunde variiert werden), die konstitutiven Widersprüche und Konflikte lösen sich aber auch hier nicht einfach auf. Sie lassen sich lediglich leichter ausblenden bzw. an die einzelnen Lehrpersonen delegieren, was aber zentrale Fragen offen hält: Wer bekommt mehr, wer weniger Aufmerksamkeit? In welchem Verhältnis stehen optimaler Lernzuwachs und Chancengleichheit? Wird ein zunehmendes Auseinanderdriften der Kompetenzen in Kauf genommen (bzw. sogar angestrebt) oder geht es zunächst um die Erreichung spezifischer Standards für alle?

- Unbeantwortet bleiben ausserdem die heiklen Fragen nach den pädagogischen Umgangsformen mit Selektionsprozessen, die mit organisierter Erziehung in komplexer Weise verknüpft sind (Luhmann \& Schorr, 1988). Die pädagogische Kritik richtet sich auf Selektionsmechanismen auf der Ebene der Organisation, bewertet diese ausschliesslich als negativ (als Aussortieren und Ausgrenzen), um in den eigenen Empfehlungen - z.B. für eine umfassende Diagnostik - dann nur noch von Förderung zu sprechen (Horstkemper, 2006). Ausgeblendet wird damit aber das Problem, dass sich 
allein über eine solche semantische Umstellung auf Förderfunktionen die Notwendigkeiten auch von Selektionsentscheidungen keineswegs einfach ausser Kraft setzen lassen - und durch Diagnose gewonnene Informationen eben nicht nur für Förderung, sondern auch für Selektion verwendet werden können bzw. sogar müssen. Noch grundsätzlicher aus dem Blickfeld gerückt wird aber die komplexe Beteiligung der Schule an gesellschaftlichen Allokationsprozessen (als Ausnahme vgl. Kronig, 2007): Was wären z.B. pädagogische Optionen für einen besseren Umgang mit Heterogenität unter gesellschaftlichen Allokationsgesichtspunkten einerseits und den Bedingungen sozialer Ungleichheit andererseits? Ginge es eher darum, für einen Ausgleich ungleicher Startchancen - im Sinne kompensatorischer, d.h. ungleicher Förderung - zu sorgen? Oder richtet sich das pädagogische Interesse eher auf die Optimierung einer leistungsbezogenen Selektion?

\section{Professionstheoretische Verkürzungen - dritte schul- theoretische Anfrage}

Ein letzter Problembereich rekurriert auf das in vielen Publikationen aufscheinende Professionsverständnis, das u. E. eng mit normativ-idealistischen Lehrerleitbildern (Baumgart \& Lange, 2008, Kap. VIII) korrespondiert: Schule, und demnach auch Lehrpersonen - so liessen sich pointiert die pädagogischen Prämissen zusammenfassen - haben sich allein am Wohle des einzelnen Kindes resp. Jugendlichen zu orientieren, unterrichten heisst ausschliesslich fördern und gute Praxis wird vor allem durch ein entsprechendes Lehrerethos garantiert.

Die Elemente eines normativ-idealistisch geprägten Professionskonzepts resultieren einerseits aus den bereits genannten pädagogischen Prämissen. Wenn nur pädagogische Aufgaben der Schule resp. des Lehrerhandelns thematisiert werden, z.B. nur über Förderung gesprochen wird, nicht aber über die eigene Beteiligung an Selektion, dann ergibt dies ein schultheoretisch verkürztes Bild der multiplen und widersprüchlichen Aufgabenstruktur des Lehrerberufs. Die etwa in strukturtheoretischen Konzepten herausgearbeiteten Antinomien und deren kompetentes Ausbalancieren - als ein Kernmerkmal des professionellen Handelns in der Schule (Helsper, 1996) - treten so erst gar nicht in den Reflexionshorizont. Andererseits setzen viele Beiträge im aktuellen Diskurs - und dies nicht allein durch ihren appellativen Modus - explizit bei der Einstellung bzw. Haltung der Lehrkräfte an, heben also berufsethische Dimensionen hervor. So geraten etwa über die «Sehnsucht nach der homogenen Lerngruppe» (Tillmann, 2008) die (falschen) pädagogischen Mentalitäten von Lehrpersonen als Reformhemmnis oder als Problemursache in den Blick, während umgekehrt die richtige Haltung eine Lösung der Probleme zu garantieren scheint: Eine Vermeidung der problematischen Selektionsmechanismen erfordere - so argumentiert Tillmann (2008, S. 77) weiter - «zuallererst eine Veränderung der pädagogischen Mentalitäten, einen Wandel in den Köpfen» (ähnlich Graumann, 2002, S. 228; Schäfers, 2009, S. 55). 
Nun sind subjektive Überzeugungen und Einstellungen zweifellos - um nicht missverstanden zu werden - eine wichtige Dimension der Lehrerprofessionalität (Sembill \& Seifried, 2009). Die entsprechenden Beschreibungen innerhalb des Reformdiskurses bleiben aber nicht nur sehr allgemein - sie kommen in der Regel nicht über das Betonen der richtigen Haltung mithilfe von Schlagwörtern hinaus. Es gibt auch hier wieder einige grundsätzliche Einwände, die bei den Appellen an eine «Veränderung der pädagogischen Mentalitäten» zu bedenken wären:

- Über die Betonung von Einstellungsfragen wird professionelles Handeln schnell auf berufsethische Aspekte verengt, und zwar bei gleichzeitiger Ausblendung anderer Dimensionen wie Wissen und Können, obwohl doch mit Blick auf die voraussetzungsreichen Ansprüche - z.B. von adaptiver Förderung (Beck et al., 2008) - durchaus zu bezweifeln wäre, ob Lehrkräfte überhaupt können, was sie sollen: Ein anderer Umgang mit Heterogenität erfordert hohe Kompetenzen, wobei auch im schulpädagogischen Diskurs alles andere als klar ist, welche spezifischen Kompetenzen dafür genau benötigt und wie sie erworben werden (können) (Trautmann \& Wischer, 2013).

- Auch wenn biografische Erfahrungen und individuelle Sichtweisen für (heterogenitätsrelevante) Einstellungen eine Rolle spielen, wäre es aus einer schul- bzw. professionstheoretischen Perspektive zu kurz gegriffen, Einstellungen allein auf Persönlichkeitsvariablen oder persönliche Vorlieben zu reduzieren: Lehrpersonen agieren in der Schule nicht als Privatpersonen, sondern ihr Handeln ist Auftragshandeln, d.h. es ist regelgeleitet und normiert (Fend, 2008). Denkt man hier nur an zentrale Lehrplanvorgaben und Prüfungsregularien, dann gibt es gute Gründe zu hinterfragen, ob etwa eine positive Wahrnehmung von Heterogenität nicht gerade durch strukturelle Bedingungen ganz erheblich eingeschränkt wird.

- Noch weiter zuspitzen lassen sich strukturelle Einflüsse schliesslich über die bereits genannten organisatorischen Prämissen: Organisationales Handeln entfaltet eine eigene Logik, die sich in institutionalisierten Erwartungen, in Verfahrensregeln, Mitgliedschaftsbedingungen und eingespielten Problemlösemechanismen ausdrückt. Und das bedeutet: Viele Entscheidungen und Handlungen sind möglicherweise weniger als ein Ausdruck individueller Normen und Überzeugungen, sondern auch als Resultat eines komplexen Abstimmungsprozesses zu deuten, der das Funktionieren des Systems aufrechtzuerhalten sucht. Ob man als einzelne Lehrperson Heterogenität wertschätzt oder auch nicht, könnte also - hier mit Gomolla und Radtke (2003) argumentiert - von durchaus untergeordneter Bedeutung sein: «Die Praxis des Erziehens in Organisationen wird bestimmt von Organisationsstrukturen, materiellen und institutionellen Vorgaben, mit denen das 
jeweilige Personal umgehen muss. (...) Erst wenn sich die Mitgliedschaftsbedingungen der Organisation ändern, wird sich auch sein Denken und Begründen umstellen können» (ebd., S. 292).

\section{Schlussbemerkung}

Unsere kritischen Überlegungen - das ist abschliessend noch einmal wichtig hervorzuheben - haben sich auf pädagogisch-normative Reflexionen als einen spezifischen Reflexionstyp innerhalb des Diskurses um Heterogenität in der Schule konzentriert. Dabei stecken dessen zentralen Merkmale wie auch die von uns herausgearbeiteten Schwachstellen schon in der von uns für diesen Typ gewählten Bezeichnung:

Einmal werden hier die komplexen Herausforderungen, die mit der Heterogenität von Schülerschaften für die Schulgestaltung verbunden sind, aus einer pädagogischen Perspektive reflektiert. Als Bezugspunkt der Beobachtung fungieren zuvorderst die Interessen und Bedürfnisse der lernenden Subjekte, was zentrale (und notwendige) Fragestellungen eröffnet: Wie kann schulisches Lernen so gestaltet werden, dass alle Schülerinnen und Schüler optimal gefördert oder Benachteiligungen von Personen bzw. Personengruppen vermieden werden? Auf welche Weise lassen sich Anerkennung und Wertschätzung von Verschiedenheit realisieren? Gleichzeitig geraten aber über diesen Fokus allein auf die "Lernerseite» die Bedingungen bzw. die "Bedürfnisse» und «Interessen» der anderen Seite - hier konkret: die Seite der Organisation - doch eher ausser Blick. Verschärft werden die daraus resultierenden Probleme durch die normative Ausrichtung als dem zweiten Merkmal des von uns betrachteten Reflexionstyps: Die Analyse der Erziehungswirklichkeit erfolgt hier nicht im Modus einer kritischen Distanz, was selbst bei dem gewählten Fokus auf die lernenden Subjekte ja durchaus möglich wäre. Die Bedürfnisse und Interessen der lernenden Subjekte werden vielmehr als zentrale Norm für die pädagogische Praxis ausgewiesen und man verfolgt zugleich das Interesse, an dieser Norm ausgerichtete Reformen anzustossen und engagiertes Handeln in der Praxis anzuregen. Ein solcher Veränderungsimpetus ist gewiss notwendig, blendet aber oft Nebenwirkungen und Unsicherheiten bezüglich der empfohlenen Alternativen aus - nicht zuletzt, weil dadurch die Überzeugungskraft und Durchsetzungsfähigkeit der eigenen Argumentation geschmälert werden könnten.

Jenseits von Fragen der normativen Anerkennung von Heterogenität, wie sie der hier vorgestellte Reformdiskurs in den Vordergrund stellt, wären deshalb in die Reformüberlegungen solche Analysen und Perspektiven deutlich stärker einzubeziehen, die das komplizierte Bedingungsgefüge, die Dynamiken, Eigenlogiken und die Begrenzungen organisierter und institutionalisierter Bildungsprozesse angemessen erfassen können. Dazu gehören ,klassische’ Reflexionsangebote der Schultheorie zur Einbindung der Schule in den gesellschaftlichen Funktions- 
zusammenhang ebenso wie organisationstheoretische Offerten. Zwar können solche Perspektiven auf Schule einerseits zu einer Begrenzung des Reformeifers und der Reformeuphorie führen, was als Verlust gedeutet werden kann. Auf der anderen Seite werden damit aber nicht nur die Grenzen, Dilemmata und transintentionalen Effekte des pädagogischen Handelns in modernen Bildungssystemen doch deutlich differenzierter erfasst, sondern auch ,realistischere' Ansatzpunkte für Reformen geliefert, die ja durchaus notwendig sind: «Erst wenn man das System versteht» - so mahnt auch Fend (2008, S. 12) «kann man eine Fehlfunktion beheben».

\section{Literatur}

Altrichter, H. \& Hauser, B. (2007). Umgang mit Heterogenität lernen. Journal für Lehrer/ innenbildung, 7 (1), 4-11.

Baumgart, F. \& Lange, U. (2008). (Hrsg.). Theorien der Schule. Erläuterungen, Texte, Arbeitsaufgaben (2. durchgesehene Auflage). Bad Heilbrunn: Klinkhardt.

Beck, E., Baer, M., Guldimann, T., Bischoff, S., Brühwiler, C., Müller, P., Niedermann, R., Rogalla, M. \& Vogt, F. (2008). Adaptive Lehrkompetenz. Analyse und Struktur, Veränderbarkeit und Wirkungen handlungssteuernden Lehrerwissens. Münster: Waxmann.

Bohl, T., Patzel, A. \& Richey, P. (2012). Öffnung - Differenzierung - Individualisierung - Adaptivität. Charakteristika, didaktische Implikationen und Forschungsbefunde verwandter Unterrichtskonzepte zum Umgang mit Heterogenität. In T. Bohl, M. Bönsch, M. Trautmann \& B. Wischer (Hrsg.), Binnendifferenzierung Teil 1: Didaktische Grundlagen und Forschungsergebnisse zur Binnendifferenzierung im Unterricht (S. 40-70). Kassel: Prolog-Verlag.

Bohnsack, F. (2008). Schule - Verlust oder Stärkung der Person? Bad Heilbrunn: Klinkhardt.

Bräu, K. \& Schwerdt, U. (2005). (Hrsg.). Heterogenität als Chance. Vom produktiven Umgang mit Gleichheit und Differenz in der Schule. Münster: Lit-Verlag.

Budde, J. (2012). Problematisierende Perspektiven auf Heterogenität als ambivalentes Thema der Schul- und Unterrichtsforschung. Zeitschrift für Pädagogik, 58 (4), 522-540.

Buholzer, A. \& Kummer-Wyss, A. (2010). (Hrsg.). Alle gleich - alle unterschiedlich! Zum Umgang mit Heterogenität in Schule und Unterricht. Seelze/Zug: Kallmeyer \& Klett.

Diederich, J. \& Tenorth, H.-E. (1997). Theorie der Schule. Ein Studienbuch zu Geschichte, Funktionen und Gestaltung. Berlin: Cornelsen.

Emmerich, M. \& Hormel, U. (2013). Heterogenität - Diversity - Intersektionalität. Zur Logik sozialer Unterscheidungen in pädagogischen Semantiken der Differenz. Wiesbaden: VS-Verlag.

Fend, H. (2008). Schule gestalten. Systemsteuerung, Schulentwicklung und Unterrichtsqualität. Wiesbaden: VS-Verlag.

Gomolla, M. \& Radtke, F.-O. (2003). Institutionelle Diskriminierung. Die Herstellung ethnischer Differenz in der Schule. Wiesbaden: VS-Verlag.

Graumann, O. (2002). Gemeinsamer Unterricht in heterogenen Gruppen. Von lernbehindert bis hochbegabt. Bad Heilbrunn: Klinkhardt.

Grossrieder, I. (2010). Gleiches und Unterschiedliches anerkennen. In A. Buholzer \& A. Kummer-Wyss (Hrsg.), Alle gleich - alle unterschiedlich! Zum Umgang mit Heterogenität in Schule und Unterricht (S. 87-96). Seelze/Zug: Kallmeyer \& Klett.

Helmke, A. \& Weinert, F. E. (1997). Bedingungsfaktoren schulischer Leistungen. In F. E. Weinert (Hrsg.). Psychologie des Unterrichts und der Schule (S. 71-176). Göttingen: Hogrefe. 
Helsper, W. (1996). Antinomien des Lehrerhandelns in modernisierten pädagogischen Kulturen. Paradoxe Verwendungsweise von Autonomie und Selbstverantwortlichkeit. In A. Combe \& W. Helsper (Hrsg.), Pädagogische Professionalität. Untersuchungen zum Typus pädagogischen Handelns (S. 521-569). Frankfurt/ Main: Suhrkamp.

Höhmann, K. (2009). Heterogenität: Eine begriffliche Klärung. In K. Höhmann, R. Kopp \& H. Schäfers (Hrsg.), Lernen über Grenzen. Auf dem Weg zu einer Lernkultur, die vom Individuum ausgeht (S. 27-35). Opladen/Farmington Hills: Barbara Budrich.

Horstkemper, M. (2006). Fördern heisst diagnostizieren. Pädagogische Diagnostik als wichtige Voraussetzung für individuellen Lernerfolg. In G. Becker, M. Horstkemper, E. Risse, L. Stäudel, R. Werning \& F. Winter (Hrsg.), Diagnostizieren und Fördern (S. 4-7). Seelze: Erhard Friedrich.

Kampshoff, M. (2009). Heterogenität im Blick der Schul- und Unterrichtsforschung. In J. Budde, \& K. Willems (Hrsg.), Bildung als sozialer Prozess. Heterogenität, Interaktion, Ungleichheiten (S. 35-52). Weinheim/München: Juventa.

Keim, W. (1977). Schulische Differenzierung: eine systematische Einführung. Köln: Kiepenheuer \& Witsch.

Klafki, W. (2002). Gesellschaftliche Funktionen und pädagogischer Auftrag der Schule in einer demokratischen Gesellschaft. In ders.: Schultheorie, Schulforschung und Schulentwicklung. Ausgewählte Studien (S. 41-62). Weinheim/Basel: Beltz.

Kronig, W. (2007). Die systematische Zufälligkeit des Bildungserfolgs - Theoretische Erklärungen und empirische Untersuchungen zur Lernentwicklung und zur Leistungsbewertung in unterschiedlichen Schulklassen. Bern/Stuttgart/Wien: Haupt.

Leschinsky, A. \& Cortina, K. S. (2005). Zur sozialen Einbettung bildungspolitischer Trends in der Bundesrepublik. In K. S. Cortina, J. Baumert, A. Leschinsky, K. U. Meyer \& L. Trommer (Hrsg.), Das Bildungswesen in der Bundesrepublik Deutschland. Strukturen und Entwicklungen im Überblick (2. Auflage, S. 20-51). Reinbeck bei Hamburg: Rowohlt.

Luhmann, N. \& Schorr, K. E. (1988). Reflexionsprobleme im Erziehungssystem. Frankfurt/ Main: Suhrkamp.

Montessori, M. (1916/1976). L'autoeducazione nelle scuole elementari. (1916; 2. Aufl. 1940; Neuausg. 1962); dt. Schule des Kindes. Montessori-Erziehung in der Grundschule. (1976).

Preisendörfer, P. (2008). Organisationssoziologie. Grundlagen, Theorien und Problemstellungen (2. Aufl.). Wiesbaden: VS-Verlag.

Prengel, A. (1993). Pädagogik der Vielfalt. Verschiedenheit und Gleichberechtigung in Interkultureller, Feministischer und Integrativer Pädagogik. Opladen: Leske \& Budrich.

Ratzki, A. (2005). Pädagogik der Vielfalt im Licht internationaler Schulerfahrungen. In Bräu, K. \& Schwerdt, U. (Hrsg.), Heterogenität als Chance. Vom produktiven Umgang mit Gleichheit und Differenz in der Schule (S. 37-52). Münster: Lit-Verlag.

Sauter, S. \& Schröder, J. (2007). Heterogenität. Eine Einführung in eine bildungswissenschaftliche Grundkategorie. Studienbrief der FernUniversität Hagen: Hagen.

Schäfers, H. (2009). Das lernende Individuum oder wie wird eigentlich gelernt? In K. Höhmann, R. Kopp, H. Schäfers \& M. Demmer (Hrsg.), Lernen über Grenzen (S. 41-67). Opladen/Farmington Hills: Barbara Budrich.

Sembill, D. \& Seifried, J. (2009). Konzeptionen, Funktionen und intentionale Veränderungen von Sichtweisen. In O. Zlatkin-Troitschanskaja, K. Beck, D. Sembill, R. Nickolaus \& R. Mulder (Hrsg.), Lehrerprofessionalität - Bedingungen, Genese, Wirkungen und Messung (S. 345-354). Weinheim: Beltz.

Tenorth, H.-E. (1987). Dogmatik als Wissenschaft - Überlegungen zum Status und zur Funktionsweise pädagogischer Argumente. In D. Baecker, J. Markowitz, R. Stichweh, H. Tyrell \& H. Wilke (Hrsg.), Theorie als Passion. Niklas Luhmann zum 60. Geburtstag (S. 694-719). Frankfurt/Main: Suhrkamp.

Terhart, E. (1986). Organisation und Erziehung. Neue Zugangsweisen zu einem alten Dilemma. Zeitschrift für Pädagogik, 32 (2), 205-224. 
Tillmann, K.-J. (2008). Viel Selektion - wenig Leistung: Erfolg und Scheitern in deutschen Schulen. In R. Lehberger \& U. Sandfuchs (Hrsg.), Schüler fallen auf. Heterogene Lerngruppen in Schule und Unterricht (S. 62-78). Bad Heilbrunn: Klinkhardt.

Trapp, E. C. (1780). Versuch einer Pädagogik. Berlin: Nicolei.

Trautmann, M. \& Wischer, B. (2011): Heterogenität in der Schule. Eine kritische Einführung. Wiesbaden: VS-Verlag.

Trautmann, M. \& Wischer, B. (2013), Professionalisierung von Lehrkräften für die Arbeit an Gemeinschaftsschulen. In T. Bohl \& S. Meissner (Hrsg.), Expertise Gemeinschaftsschule. Forschungsergebnisse und Handlungsempfehlungen für Baden-Württemberg (S. 47-59). Weinheim/Basel: Beltz.

Wischer, B. (2009). Der Diskurs um Heterogenität und Differenzierung. Beobachtungen zu einem schulpädagogischen 'Dauerbrenner'. In B. Wischer \& K.-J. Tillmann (Hrsg.), Erziehungswissenschaft auf dem Prüfstand. Schulbezogene Forschung und Theoriebildung von den 1970er Jahren bis heute (S. 69-96). Weinheim/München: Juventa.

Wischer, B. (2013). Konstruktionsbedingungen von Heterogenität im Kontext organisierter Lernprozesse. Eine schul- und organisationstheoretische Problemskizze. In J. Budde (Hrsg.), Unscharfe Einsätze. (Re-)Produktion von Heterogenität im schulischen Feld (S. 99-126). Wiesbaden: VS-Verlag.

Zymek, B. (2011). Welche Homogenität und welche Heterogenität soll das Schulsystem gewährleisten? Historische und aktuelle, nationale und internationale Befunde. In R. Schilmöller \& C. Fischer (Hrsg.), Heterogenität als Herausforderung für schulisches Lernen (S. 83-94). Münster: Aschendorff.

Schlagworte: Reformdiskurs zu Heterogenität, Lehrerprofessionalität, Differenzierung, Schule als Institution und Organisation, Heterogenitätskonzepte

\section{Questions théoriques relatives au débat pédagogico-normatif sur l'hétérogénéité scolaire}

\section{Résumé}

Cet article propose une analyse critique des réflexions pédagogico-normatives émises dans le débat actuel sur l'hétérogénéité scolaire. Une typologie de réflexions considérées fondamentales est d'abord proposée, afin de discuter ensuite trois problèmes qui en émergent: (1) l'imprécision et la sur-complexité de la notion d'hétérogénéité, résultant d'une pensée centrée sur l'enfant en tant qu'individu; (2) l'occultation de la logique structurelle et fonctionnelle de l'apprentissage institutionnalisé, et (3) des théories professionnelles réductrices fondées sur des modèles d'enseignement pédagogico-normatifs.

Mots-clés: Débat sur l'hétérogénéité, professionnalisme de l'enseignant, différenciation, école en tant qu'institution et organisation, concepts d'hétérogénéité 


\section{Domande di teoria scolastica relative al dibattito sull'eterogeneità pedagogico-normativa}

\section{Riassunto}

L'articolo punta a un confronto critico con riflessioni pedagogico-normative nell'attuale dibattito sull'eterogeneità. Inizialmente si ricostruisce la tipologia di riflessione alla base, che funge da punto di partenza per la discussione di tre problemi selezionati che emergono dalla riflessione stessa: (1) una mancata distinzione e un'eccessiva complessità del concetto di eterogeneità che risulta dal pensiero del singolo bambino; (2) l'esclusione delle logiche strutturali e funzionali dell'apprendimento istituzionalizzato e (3) una riduzione teorico-professionale attraverso modelli di insegnamento normativo-pedagogici.

Parole chiave: Dibattito riformista sull'eterogeneità, professionalità degli insegnanti, differenziazione, scuola come istituzione e organizzazione, filosofia dell'eterogeneità

\section{Critical Remarks on the Normative Discourse about School Heterogeneity}

\section{Summary}

This article aims at challenging pedagogical-normative reflexions within the current discourse on school heterogeneity. After reconstructing the underlying mode of discourse argumentation, three problems are discussed: (1) vagueness and overcomplexity of the notion of heterogeneity, resulting from child-centered pedagogy, (2) neglect of structural and functional aspects of institutionalized learning, and (3) normative-pedagogical concepts of teaching that appear oversimplified in the light of professional theory.

Key words: Normative discourse on student diversity, teacher professionalism, differentiation, school as institution and organization, concepts of heterogeneity 